\title{
The fellow eye of patients with phakic rhegmatogenous retinal detachment from atrophic holes of lattice degeneration without posterior vitreous detachment
}

\author{
C R Gonzales, A Gupta, S D Schwartz, A E Kreiger
}

Br J Ophthalmol 2004;88:1400-1402. doi: 10.1136/bjo.2004.043240

Background: Primary phakic rhegmatogenous retinal detachment (RRD) without posterior vitreous detachment (PVD) represents a unique clinical entity that behaves differently from RRD associated with PVD. While previous studies have reported the long term findings in the fellow eye of patients with RRD and PVD, the outcome of the fellow eye of patients with RRD without PVD is not known.

Methods: Consecutive patients with RRD not associated with PVD were studied retrospectively. The authors evaluated the fellow eye for retinal detachment or other vision threatening pathology.

Results: 27 patients (mean age 32 years) were studied with follow up of between 9 and 326 months (mean 111 months). $24(89 \%)$ were myopic. Bilateral retinal detachment occurred in eight patients $(30 \%)$. On initial examination, 17 patients (63\%) had retinal findings (including lattice degeneration, atrophic holes, and/or cystic retinal tufts) in the fellow eye that might predispose them to retinal detachment. 14 vision threatening events or diagnoses occurred (nine of which were rhegmatogenous in nature) in the fellow eye including eight retinal detachments, one traumatic PVD without retinal tears, one retinal tear after PVD, one diagnosis of pigmentary glaucoma needing trabeculectomy, two visually significant cataracts, and one diagnosis of chorioretinitis. 23 patients (85\%) maintained visual acuity better than 20/50, with most retaining 20/20 vision in the fellow eye.

Conclusions: Patients who experience RRD without PVD are at risk of developing vision threatening events in the contralateral eye and, as such, the fellow eye should be followed carefully.

$\mathrm{T}$ wo distinct types of retinal detachment have been described in association with lattice degeneration-those associated with tractional tears at the border of lattice lesions and those associated with round atrophic holes. ${ }^{12}$ Retinal detachments due to atrophic holes in lattice tend to be indolent and occur frequently in younger, myopic patients. ${ }^{2-5}$ This type of retinal detachment often occurs in the absence of a posterior vitreous detachment (PVD), which probably accounts for its indolent, often asymptomatic nature. Degenerative retinal lesions, such as lattice degeneration and atrophic holes, often occur bilaterally and may predispose the fellow eye to retinal detachment. ${ }^{67}$

Previous studies of phakic retinal detachment due to atrophic holes of lattice degeneration have reported retinal detachment to occur in 5-12\% of fellow eyes. ${ }^{7-9}$ Most studies of retinal detachment from atrophic holes do not address the status of the vitreous or they have included patients with and without PVD. We studied patients with primary phakic
"non-PVD related" retinal detachment to determine the frequency of vision threatening events including "clinical" retinal detachment requiring surgery and ocular findings predisposing to retinal detachment in the fellow eye.

\section{PATIENTS AND METHODS}

Institutional review board (IRB)/ethics committee approval was obtained for this study. All patients consecutively diagnosed with clinical rhegmatogenous retinal detachment (RRD) requiring surgical repair between March 1970 and April 1999 were identified from the clinical database of a single surgeon (AEK) at the Jules Stein Eye Institute. Only patients with primary phakic retinal detachment without associated PVD, those causally related to atrophic holes of lattice degeneration, were studied. Patients with less than 6 months of follow up were excluded. When appropriate follow up was lacking, the patient or the patient's ophthalmologist was contacted to retrieve further information. A thorough chart review was performed and the relevant clinical data were recorded.

\section{RESULTS}

In all, 434 patients were identified with the diagnosis of primary RRD. Of these, 31 were determined by review of the medical record to have primary phakic retinal detachment without PVD associated with atrophic holes of lattice degeneration. Four were excluded because of insufficient follow up. Of the 27 remaining patients, nine women and 18 men, the ages ranged between 16 and 53 years of age (mean 32 years). The follow up interval was between 9 and 326 months (mean 111 months, median 76 months).

The findings on initial examination are recorded in table 1 for both eyes. Six patients $(22 \%)$ had previous or newly diagnosed retinal detachment in the fellow eye at the time of initial examination. All three newly diagnosed retinal detachments were related to atrophic holes of lattice degeneration without PVD; all three underwent surgical repair with scleral buckling and remained attached throughout the follow up period. Of the remaining 21 patients without retinal detachment, 18 (86\%) had some ocular finding predisposing them to retinal detachment, including lattice degeneration, high myopia, and/or cystic retinal tufts.

Two patients later developed non-PVD related retinal detachment, which was successfully repaired with scleral buckling procedure, giving an overall incidence of retinal detachment in the fellow eye of $30 \%$. Fourteen vision threatening events occurred in the fellow eye including eight retinal detachments (six of which were diagnosed before or concurrent with the initial examination), one traumatic

Abbreviations: PVD, posterior vitreous detachment; RRD, rhegmatogenous retinal detachments 
Table 1 Findings on initial ocular examination

\begin{tabular}{lcl}
\hline & Primary eye & Fellow eye \\
\hline High myopia* & 12 & 13 \\
Low myopia & 11 & 11 \\
Lattice degeneration & 27 & 16 \\
With atrophic holes & 27 & 10 \\
Without holes & 0 & 6 \\
Resolved RD & 0 & 1 \\
Primary phakic RD & 27 & $3 \dagger$ \\
S/p scleral buckle procedure & 0 & 2 \\
S/p laser retinopexy & 1 & 0 \\
Cystic retinal tuft & 1 & 2 \\
\hline
\end{tabular}

$R D$, retinal detachment; $S / p$, status post.

*High myopia was defined as $>-6.00 \mathrm{D}$; low myopia was defined as $\leqslant-6.00 \mathrm{D}$.

†All 3 patients were successfully treated with scleral buckling procedure.

posterior vitreous detachment without retinal tears, one retinal tear after PVD (treated with laser), two visually significant cataracts, one diagnosis of pigmentary glaucoma needing trabeculectomy, and one diagnosis of chorioretinitis. These diagnoses are listed in table 2. Nine of $14(64 \%)$ events were rhegmatogenous in nature.

The visual acuity data are presented in table 3. Eighty five per cent of patients retained visual acuity better than 20/50 in the fellow eye, with $74 \%$ retaining $20 / 20$ vision. Of the three patients with $\leqslant 20 / 50$ vision, two were secondary to cataract, and one was secondary to maculopathy from previous retinal detachment.

A scleral buckling procedure was performed in 26/27 patients $(96 \%)$ in the primary eye with $96 \%$ success after one surgery; one patient was observed without progression. One patient needed subsequent removal of an infected scleral buckle, and the retina remained attached. Recurrent retinal detachment with proliferative vitreoretinopathy occurred in one patient; it was successfully repaired with scleral buckle revision.

\section{DISCUSSION}

Retinal detachment due to atrophic holes of lattice degeneration has been well described. Byer reported a 43\% incidence of round atrophic holes in patients with lattice degeneration of the retina; clinical or progressive subclinical retinal detachment occurred in $2 \%$ of these eyes. ${ }^{4}$ Previous studies investigating the fellow eye in patients with phakic retinal detachments associated with atrophic holes of lattice degeneration have reported bilaterality of $12-39 \% .{ }^{17-9}$ Although these detachments usually occur in the absence of a PVD, the nature of the vitreous was not always specified or patients with and without PVD were included in many of these studies. All patients in this series had retinal detachment due to atrophic holes in lattice degeneration without an associated PVD in the primary eye.

Thirty per cent of the patients developed retinal detachment in the fellow eye during the follow up interval averaging 111 months, and all but one required surgical repair. Detailed drawings of the retinal detachment in the fellow eye were available for review in five of these eight patients, and all were associated with atrophic holes of lattice without PVD. The incidence of bilaterality of retinal detachment in our study was twice as high as that found in two other studies of retinal detachment from atrophic holes of lattice; both studies found bilateral retinal detachment in $12 \%$ of patients. ${ }^{78}$ Unlike these authors, we chose to include patients with a history of retinal detachment in the fellow eye to accurately determine the incidence of bilaterality, which explains why we found a higher incidence than previously reported.
Table 2 Vision threatening events/diagnoses during follow up in the fellow eye

\begin{tabular}{ll}
\hline Event/new diagnosis & No \\
\hline Visually significant cataract & 2 \\
PVD with retinal tear & 1 \\
Rhegmatogenous retinal detachment & 2 \\
PVD without retinal tear & 1 \\
Pigmentary glaucoma requiring surgery & 1 \\
Chorioretinitis & 1 \\
Total & 8 \\
\hline PVD, posterior vitreous detachment. &
\end{tabular}

Table 3 Final visual acuity

\begin{tabular}{lcc}
\hline Final visual acuity & First eye & Fellow eye \\
\hline $20 / 20$ & 21 & 20 \\
$20 / 25-20 / 40$ & 3 & 3 \\
$20 / 50-20 / 100$ & 2 & 2 \\
$20 / 200$ & 1 & 1 \\
$<20-400$ & 0 & 0 \\
Total no of eyes & 27 & 26 \\
\hline
\end{tabular}

The status of the fellow eye is important to establish upon initial examination since predisposing lesions or concurrent retinal detachment may be detected. Atrophic retinal breaks in areas of lattice degeneration and cystic retinal tufts are findings that may predispose to retinal detachment, and these lesions may cause retinal breaks in eyes lacking PVD. ${ }^{10}$ Sixty three per cent of patients in this series had one or more predisposing retinal lesion in the fellow eye noted on initial examination, with lattice degeneration present in 16/27 patients $(60 \%)$. These findings are similar to those from other studies. $^{78}$

Four patients in this study received laser retinopexy or cryotherapy for lattice degeneration with or without retinal holes. These treatments were done during a time when laser retinopexy for lattice degeneration was frequently performed. It is beyond the scope of this study to determine whether or not this treatment was beneficial in preventing retinal detachment in these patients who received prophylactic treatment.

None of the patients in this study had pre-existing PVD in the fellow eye, and only two developed PVD during the follow up period, which is not surprising given the relatively young age of the patient population. One of these patients developed a tractional tear without detachment in an eye that was previously buckled, and one patient developed a PVD without retinal tears after trauma in a previously buckled eye.

While previous studies have shown retinal tears in 20-21\% of fellow eyes with retinal detachments, these studies included patients with tractional tears from PVD in the primary eye. ${ }^{6}{ }^{11}$ In our study, only one patient (4\%) developed a retinal tear in the fellow eye that was not present upon initial examination. This discrepancy is not surprising given the absence of PVD in the fellow eyes of this study.

Other investigators have demonstrated favourable prognoses in patients with retinal detachment due to lattice degeneration in terms of reporting less PVR and better visual outcomes. ${ }^{3}$ We also noted excellent visual outcomes, with most patients maintaining 20/20 vision. Three patients had vision worse than 20/40 in the fellow eye, two from cataract and one from previous macular detachment. The other nonrhegmatogenous events, chorioretinitis and pigmentary glaucoma, were not associated with visual loss. 
Phakic retinal detachment from atrophic holes within areas of lattice degeneration has been shown to occur in younger patients and in those with more myopic refractive error. ${ }^{138}$ Our study also demonstrated such trends with the mean age of 32 years and $48 \%$ of the patients having greater than $-6.00 \mathrm{D}$ of myopia.

This study is limited by its retrospective nature. Additionally, the patients were identified from a single surgeon's practice and were seen at a tertiary referral centre, which may bias our findings. On the other hand, the study is strengthened by detailed retinal drawings with special attention to the presence or absence of PVD in all cases. All patients were followed for a minimum of 6 months with an overall average follow up of 9.3 years.

In summary, round atrophic holes in lattice degeneration are an important cause of phakic retinal detachments. These detachments tend to occur in younger myopic patients. These patients with retinal detachment in the absence of PVD are at considerable risk of developing detachment of the fellow eye. They frequently exhibit retinal findings that may predispose them to retinal detachment, and should have careful examination and follow up.

\section{Authors' affiliations}

C R Gonzales, A Gupta, S D Schwartz, A E Kreiger, Jules Stein Eye Institute, Department of Ophthalmology, University of California, UCLA David Geffen School of Medicine, Los Angeles, CA, USA
Presented at the Retina Congress Combined Meeting, 2 October 2002.

Correspondence to: Christine Read Gonzales, MD, Jules Stein Eye Institute, 100 Stein Plaza, Los Angeles, CA 90095, USA; gonzales@jsei. ucla.edu

Accepted for publication 17 March 2004

\section{REFERENCES}

1 Malbran ES, Dodds R, Hulsbus R. Two distinct types of myopic retinal detachment. Mod Probl Ophthalmol 1977; 18:292-303

2 Morse PH. Lattice degeneration of the retina and retinal detachment. Am J Ophthalmol 1974;78:930-4.

3 Benson WE, Morse PH. The prognosis of retinal detachment due to lattice degeneration. Ann Ophthalmol 1978;Sept: 1197-200.

4 Byer NE. Lattice degeneration of the retina. Surv Ophthalmol 1979;23:213.

5 Byer NE. What happens to untreated asymptomatic retinal breaks, and are they affected by posterior vitreous detachment? Ophthalmology 1998;105:1045-50.

6 Everett WG. The fellow eye syndrome in retinal detachment. Am J Ophthalmol 1963;56:739.

7 Tillery WV, Lucier AC. Round atrophic holes in lattice degeneration - an important cause of phakic retinal detachment. Trans Am Acad Ophthalmol Otol 1976;81:509-18.

8 Murakami-Nagasako F, Ohba N. Phakic retinal detachment associated with atrophic holes of lattice degeneration of the retina. Graefes Arch Clin Exp Ophthalmol 1983;220:175-8.

9 Folk JC, Arrindell EL, Klugman MR. The fellow eye of patients with phakic lattice retinal detachment. Ophthalmology 1989;96:72-9.

10 Foos RY. Retinal holes. Am J Ophthalmol 1978;86:354.

11 Lorentzen SE. Frequency of lattice degeneration in the fellow eye in retinal detachment. Acta Ophthalmol 1988;66:157-60. 